\title{
ON THE SHAPE OF INVADING POPULATION IN ANISOTROPIC ENVIRONMENTS
}

\author{
VIKTORIA BLAVATSKA*
}

\begin{abstract}
We analyze the properties of population spreading in environments with spatial anisotropy within the frames of a lattice model of asymmetric (biased) random walkers. The expressions for the universal shape characteristics of the instantaneous configuration of population, such as asphericity $A$ and prolateness $S$ are found analytically and proved to be dependent only on the asymmetric transition probabilities in different directions. The model under consideration is shown to capture, in particular, the peculiarities of invasion in presence of an array of oriented tubes (fibers) in the environment.
\end{abstract}

Mathematics Subject Classification. 60G50, 62P10, 65C60.

Received May 22, 2019. Accepted October 19, 2019.

\section{INTRODUCTION}

The problem of spreading of a population of agents in anisotropic environments with some preferred orientations of movement is encountered in a rich variety of biological phenomena and medicine. In general, the movement characteristics are influenced due to the environmental heterogeneity. Important examples include behavior of chemosensitive cells like bacteria or leukocytes in the gradient of a chemotactic factor [2, 9, 14, 22], the motion of micro-organisms under the action of gravitational force (gravitaxis) or a light source (phototaxis) $[27,35,49]$, the cell migration in fiber network of extracellular environment $[15,19,20,28,36]$ and in particular the tumor invasion and metastasis in the tissue matrix $[1,3,25]$. The important problems of spatial ecology are connected with analysis of migrations of living organisms in oriented habitats with orientation given by magnetic cues, elevation profiles, spatial distributions of resources [5, 18, 29]. The variations of above characteristics has non-trivial influence on the population growth, persistence and dispersal [5, 13, 30, 51]. In particular, the presence of oriented factors in environment lead to occurrence of directed movement patterns, different from pure diffusion $[4,7,13]$. In this concern, it is worthwhile to mention also the modern technologies of controlled drug delivery, using an external oriented magnetic field [54], and the implants based on arrays of oriented $\mathrm{TiO}_{2}$ nanotubes, which control the directed release of drugs $[32,50]$.

The model of a random walk (RW) on a regular lattice provides a good description of the stochastic processes [46]. In the simplest case, when there is no preferred direction, this process restores the Brownian motion and such a model may be shown to produce the standard diffusion equation. Making the probabilities of moving in different directions not equal causes the directional bias, which leads to the drift-diffusion equation. Such

Keywords and phrases: Random walk, heterogeneous environment, biological invasion.

Department for Computer Simulations of Many-Particle Systems, Institute for Condensed Matter Physics of the National Academy of Sciences of Ukraine, Lviv 79011, Ukraine.

* Corresponding author: blavatskav@gmail.com 
asymmetric biased random walks (BRW) are frequently used in biology to model the motion of living organisms and cells in oriented environments [16,37]. The bias may be caused both by the fixed external environmental factors (such as gravitational force or external magnetic field), and by varying factors (such as chemical gradient or food resources in oriented migration of organisms). Thus, the transition probabilities in BRW model can also be not only constants, but also functions of space and time.

The problem of determining the size and shape of individual random walk trajectory, treated as track of a particle (cell) in environment, attracts a considerable attention of researchers. In the pioneering paper of Kuhn [31] it was suggested, that RW trajectory is a highly aspherical object. In the following studies [47, 48] the averaged principal components of inertia tensor were introduced as parameters for description of RW asymmetry. Later in references $[6,39]$ it was proposed to characterize the shape properties by the set of rotationally invariant combinations of inertia tensor components, such as asphericity $A$ and prolateness $S$. These shape characteristics of RW trajectories were estimated both analytically [21, 26, 42, 53] and numerically [11].

From the point of view of biological application, an importance of analyzing the tracks of single cells can be realized e.g. in the processes of the guidance of dendritic cells by haptotactic chemokine gradients towards lymphatic vessels [52] or for neutrophil migration directed by inflammatory chemokines [41]. In particular, the neutrophil migration appeared to be of random walk type with directed track segments induced by chemokine gradients. The universal shape parameters, such as asphericity of individual cell tracks were evaluated in [34] making use of image data for synthetic cells and in vitro neutrophil tracks, obtained in microscopy experiments.

Processes of bacteria growing and invasion are known to produce colonies of various shapes, called "patterns" or "morphotypes" $[23,38,45]$. Colony patterns serve for differentiation of populations of individuals otherwise identical. The shape of a bacterial colony depends on variables like the nutrient diffusion field, cyclic production of chemoattractants and chemorepellents, long range chemical signalling such as quorum sensing and production of secreted wetting fluid [8]. The significance of analyzing the bacterial colony patterns resides in a deeper understanding of colony evolution and morphogenesis in the given environment.

Different technologies like genetic engineering and histochemical staining [43], scanning electron microscopy [44] etc. are applied to investigate the geometry of growth and invasion of bacteria colonies. The asymmetric shape pattern formation is found e.g. when colonies encountered obstacles in substrate during development, such as glass fibers or other bacteria co-existing [38, 45] or chemical fields [40]. Formation of patterns of unusual shape can also signalize about arising of mutations in given population [23, 33]. Processes of cell migration in disordered extracellular environment and tissue matrices $[1,15,19,20,25,28,36]$ also lead to formation of asymmetrical shape patterns of cell populations. The geometrical characterisitcs of animal groups are also of interest, e.g. the impact of anisotropic interactions between individuals on the shape of groups have been analyzed recently in [17].

In this concern, it is worthwile to expose the asphericity $A$ and prolateness $S$ as shape characteristics of an instantaneous configuration of a group of particles, spreading in anisotropic environment with preferred orientations. These shape parameters may be of use in mathematical biology: they enable to classify the shape patterns, formed in processes of bacteria growing in inhomogeneous substrates or cell invasion in extracellular matrix of connective tissues with collagen and elastin fibers. Since the time-dependent positions of cells or bacteria in process of migrations are possible to record in scanning microscopy experiments, these geometrical shape characteristics can be directly measurable by analyzing the corresponding image data by statistical means. Note that recently the parameters $A$ and $S$ were used to describe the instantaneous shapes of clouds of particle in turbulent flows in reference [10], where it was observed an increase of asphericity of such clouds under the action of oriented external fields.

In the present work, we exploit the lattice model of BRW spreading in anisotropic environment with preferred orientations. We aim to express the shape characterisitcs $A$ and $S$ in terms of fundamental random walk parameters (such as transition probabilities) and analyze how the presence of orientational factors and heterogeneity in environment impact the geometrical shape of an instantaneous configuration of group of random walkers.

As it will be shown, such a model allows us in particular, to analyze the spreading process in presence of structural inhomogeneities in form of oriented lines. In particular, such a model resembles the arrays of oriented nanotubes in controlled drug delivery implants, mentioned above. 
The layout of the paper is as follows. In the next section, we introduce the model and define the observables we are interested in. The analytical expressions of shape parameters are given in Section 3, followed by examples of some model cases presented in Section 4. We end up by giving conclusions and outlook.

\section{THE MODEL}

We start with considering a population of $N$ random walkers, spreading on an infinite 3 -dimensional lattice. At each time step, each the walker jumps towards one of 6 nearest neighbor sites with corresponding probability $p_{i}, i=1, \ldots, 6$ such that $\sum_{i=1}^{6} p_{i}=1$ (as schematically shown on Fig. 1). In the simplest case of isotropic uncorrelated random walk, all the transition probabilities are equal: $p_{i}=\frac{1}{6}$. We assume, that all the walkers start to move from the same starting point (let it be the center of coordinate system), so that we start with highly dense configuration ("drop"), localized in space. The walkers move independently, without any interactions or correlations between them.

The probability $P\left(t,\left\{n_{i}^{a}\right\}\right)$ that the walker $a$ had performed $n_{i}^{a}$ steps in directions $i(i=1, \ldots, 6)$ after the total amount of $t$ steps (so that $t=\sum_{i=1}^{6} n_{i}^{a}$ ) is given by:

$$
P\left(t,\left\{n_{i}^{a}\right\}\right)=\frac{t !}{\prod_{i=5}^{5} n_{i}^{a} !\left(t-\sum_{i=1}^{5} n_{i}^{a}\right) !} \prod_{i=1}^{6} p_{i}^{n_{i}^{a}},
$$

with $\sum_{\left\{n_{i}^{a}\right\}} P\left(t,\left\{n_{i}^{a}\right\}\right)=1$.

Thus, we can define the configurational averaging of any observable $\mathcal{O}$ over all possible trajectories of particle $a$ at time $t$ accordingly to:

$$
\langle\mathcal{O}\rangle=\mathcal{O} P\left(t,\left\{n_{i}^{a}\right\}\right)
$$

To evaluate e.g. the mean value of $\left\langle n_{1}^{a}\right\rangle$, we first "sum out" the remaining $n_{i}^{a}$ with $i>1$ in equation (2.1) according to:

$$
\begin{array}{r}
P\left(t, n_{1}^{a}\right)=\sum_{n_{2}^{a}=0}^{t-n_{1}^{a}} \cdots \sum_{n_{5}^{a}=0}^{t-n_{1}^{a}-n_{2}^{a}-n_{3}^{a}-n_{4}^{a}} \\
P\left(t,\left\{n_{i}^{a}\right\}\right)==\frac{t !}{n_{1}^{a} !\left(t-n_{1}^{a}\right) !} p_{1}^{n_{1}^{a}}\left(1-p_{1}\right)^{t-n_{1}^{a}},
\end{array}
$$

so that:

$$
\left\langle n_{1}^{a}\right\rangle=\sum_{n_{1}^{a}=0}^{t} n_{1}^{a} P\left(t, n_{1}^{a}\right)=t p_{1},
$$

and in general:

$$
\begin{aligned}
& \left\langle n_{i}^{a}\right\rangle=\sum_{n_{i}^{a}=0}^{t} n_{i}^{a} P\left(t, n_{i}^{a}\right)=t p_{i}, \\
& \left\langle\left(n_{i}^{a}\right)^{2}\right\rangle=\sum_{n_{i}^{a}=0}^{t}\left(n_{i}^{a}\right)^{2} P\left(t, n_{i}^{a}\right)=t p_{i}+t(t-1) p_{i}^{2}, \\
& \left\langle n_{i}^{a} n_{j}^{a}\right\rangle=\sum_{n_{i}^{a}=0}^{t} \sum_{n_{j}^{a}=0}^{t-n_{i}^{a}} n_{i}^{a} n_{j}^{a} P\left(t, n_{i}^{a}, n_{j}^{a}\right)=t(t-1) p_{i} p_{j}, i \neq j,
\end{aligned}
$$




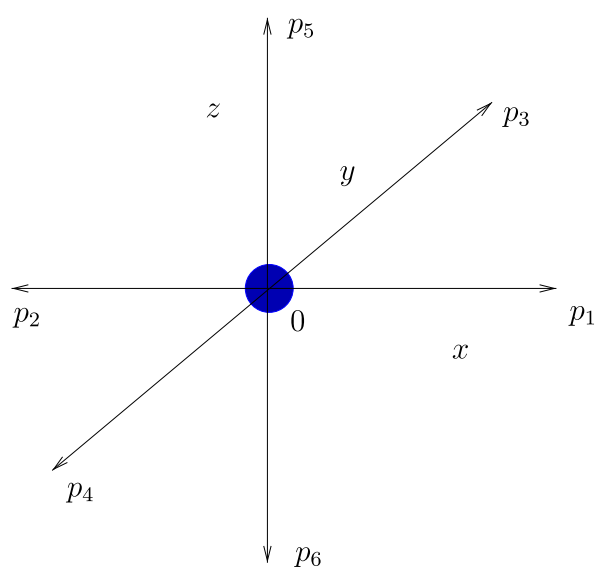

FiguRE 1. Schematic presentation of a random walk process. The particle is putted on a site of a regular 3-dimensional lattice and at each time step makes a jump towards one of possible 6 directions with corresponding probability $p_{i}(i=1, \ldots, 6)$.

in the last equation, $P\left(t, n_{i}^{a}, n_{j}^{a}\right)$ is obtained by summing out all $n_{k}^{a}$ with $k \neq i$ and $k \neq j$ in the same way as in equation (2.3).

Since we assume, that walkers $a$ and $b$ move independently, we also have:

$$
\left\langle n_{i}^{a} n_{j}^{b}\right\rangle=\sum_{n_{i}^{a}=0}^{t} n_{i}^{a} P\left(t, n_{i}^{a}\right) \sum_{n_{j}^{b}=0}^{t} n_{j}^{b} P\left(t, n_{j}^{b}\right)=t^{2} p_{i} p_{j} .
$$

\section{Results: universal SHAPE PARAMETERS}

Let $\vec{R}_{a}(t)=\left\{x^{a}(t), y^{a}(t), z^{a}(t)\right\} \equiv\left\{x_{1}^{a}(t), x_{2}^{a}(t), x_{3}^{a}(t)\right\}$ be the position vector of the $a$ th walker at time $t$ $(a=1, \ldots, N)$. The position of each walker, averaged over an ensemble of all possible trajectories of particle, can be easily obtained using the results of previous Section. Really, since e.g. the averaged coordinate $\left\langle x_{1}(t)\right\rangle$ of a walker is given by a difference of number of steps to the right and to the left along the $x_{1}$-axis, we have:

$$
\begin{aligned}
& \left\langle x_{1}^{a}(t)\right\rangle=\left\langle n_{1}^{a}-n_{2}^{a}\right\rangle=t\left(p_{1}-p_{2}\right), \\
& \left\langle x_{2}^{a}(t)\right\rangle=\left\langle n_{3}^{a}-n_{4}^{a}\right\rangle=t\left(p_{3}-p_{4}\right), \\
& \left\langle x_{3}^{a}(t)\right\rangle=\left\langle n_{5}^{a}-n_{6}^{a}\right\rangle=t\left(p_{5}-p_{6}\right),
\end{aligned}
$$

so that: $\left\langle x_{i}^{a}(t)\right\rangle=t\left(p_{2 i-1}-p_{2 i}\right)$.

Correspondingly:

$$
\begin{aligned}
& \left\langle\left(x_{i}^{a}(t)\right)^{2}\right\rangle=t\left(p_{2 i-1}+p_{2 i}\right)+t(t-1)\left(p_{2 i-1}-p_{2 i}\right)^{2}, \\
& \left\langle x_{i}^{a}(t) x_{j}^{a}(t)\right\rangle=t(t-1)\left(p_{2 i-1}-p_{2 i}\right)\left(p_{2 j-1}-p_{2 j}\right), i \neq j \\
& \left\langle x_{i}^{a}(t) x_{i}^{b}(t)\right\rangle=t^{2}\left(p_{2 i-1}-p_{2 i}\right)^{2}, \\
& \left\langle x_{i}^{a}(t) x_{j}^{b}(t)\right\rangle=t^{2}\left(p_{2 i-1}-p_{2 i}\right)\left(p_{2 j-1}-p_{2 j}\right) .
\end{aligned}
$$


The shape properties of configuration of the population can be characterized [6,39] in terms of the gyration tensor $\mathbf{Q}$ with components:

$$
\left\langle Q_{i j}(t)\right\rangle=\frac{1}{N(N-1)} \sum_{\substack{a, b=1 \\ a<b}}^{N}\left\langle\left(x_{i}^{a}(t)-x_{i}^{b}(t)\right)\left(x_{j}^{a}(t)-x_{j}^{b}(t)\right)\right\rangle, \quad i, j=1,2,3 .
$$

The spread in eigenvalues $\lambda_{i}(t)(i=1,2,3)$ of the gyration tensor describes the distribution of particles in configuration and thus measures the asymmetry of a shape. In particular, in completely isotropic symmetric case all the eigenvalues $\lambda_{i}(t)$ are equal.

Let us introduce the rotationally invariant universal combinations of components of the gyration tensor $[6,39]$. Let $\lambda_{\mathrm{av}}(t) \equiv \operatorname{Tr} \mathbf{Q} / 3$ be the average eigenvalue of the gyration tensor. Then the extent of asphericity of an instantaneous configuration of population is characterized by quantity $A$ defined as:

$$
\begin{aligned}
A= & \frac{1}{6} \sum_{i=1}^{3} \frac{\left(\lambda_{i}(t)-\lambda_{\mathrm{av}}(t)\right)^{2}}{\lambda_{\mathrm{av}}(t)^{2}}=\left(\sum_{i=1}^{3}\left\langle Q_{i i}(t)\right\rangle^{2}+3 \sum_{\substack{i, j=1 \\
i<j}}^{3}\left\langle Q_{i j}(t)\right\rangle^{2}\right. \\
& \left.-\sum_{\substack{i, j=1 \\
i<j}}^{3}\left\langle Q_{i i}(t)\right\rangle\left\langle Q_{j j}(t)\right\rangle\right) /\left(\sum_{i=1}^{3}\left\langle Q_{i i}(t)\right\rangle\right)^{2} .
\end{aligned}
$$

This universal quantity equals zero for a completely isotropic spherical configuration, where all the eigenvalues are equal $\lambda_{i}=\bar{\lambda}$, and takes a maximum value of one in the case of a stretched highly anisotropic configuration, where all the eigenvalues equal zero except of one. Thus, the inequality holds: $0 \leq A \leq 1$. Another rotationally invariant quantity, defined in three dimensions, is the so-called prolateness $S$ :

$$
\begin{aligned}
S= & \frac{\prod_{i=1}^{3}\left(\lambda_{i}(t)-\lambda_{\mathrm{av}}(t)\right)}{\lambda_{\mathrm{av}}(t)^{3}}=\left(2 \sum_{i=1}^{3}\left\langle Q_{i i}(t)\right\rangle^{3}-3 \sum_{\substack{i, j=1 \\
i \neq j}}^{3}\left\langle Q_{i i}(t)\right\rangle^{2}\left\langle Q_{j j}(t)\right\rangle\right. \\
& -18 \sum_{\substack{i, j, k=1 \\
i \neq j \neq k}}^{3}\left\langle Q_{i i}(t)\right\rangle\left\langle Q_{j k}(t)\right\rangle^{2}+9 \sum_{\substack{i, j=1 \\
i \neq j}}^{3}\left\langle Q_{i i}(t)\right\rangle\left\langle Q_{i j}(t)\right\rangle^{2} \\
& \left.+12 \prod_{i=1}^{3}\left\langle Q_{i i}(t)\right\rangle+54\left\langle Q_{12}(t)\right\rangle\left\langle Q_{13}(t)\right\rangle\left\langle Q_{23}(t)\right\rangle\right) /\left(\sum_{i=1}^{3}\left\langle Q_{i i}(t)\right\rangle\right)^{3} .
\end{aligned}
$$

For absolutely prolate, stretched rod-like configuration $\left(\lambda_{1} \neq 0, \lambda_{2}=\lambda_{3}=0\right)$, the parameter $S$ equals two, whereas for absolutely oblate, disk-like shape $\left(\lambda_{1}=\lambda_{2}, \lambda_{3}=0\right)$ it takes on a value of $-1 / 4$. In general, $S$ is positive for prolate ellipsoid-like shape $\left(\lambda_{1} \gg \lambda_{2} \approx \lambda_{3}\right)$ and negative for oblate ones $\left(\lambda_{1} \approx \lambda_{2} \gg \lambda_{3}\right)$, whereas its magnitude measures how oblate or prolate the configuration is.

Next, we will find the exact values of quantities (3.7) and (3.8) for a system of non-interacting $N$ asymmetric random walkers on a lattice.

Expressions (3.2)-(3.5) allow us to find the averaged components of gyration tensor. For example:

$$
\begin{aligned}
\left\langle Q_{11}(t)\right\rangle & =\frac{1}{N(N-1)}\left\langle\sum_{\substack{a, b=1 \\
a<b}}^{N}\left(x_{1}^{a}(t)-x_{1}^{b}(t)\right)^{2}\right\rangle \\
& =\left\langle\left(x_{1}^{a}(t)\right)^{2}\right\rangle-\left\langle x_{1}^{a}(t) x_{1}^{b}(t)\right\rangle=t\left(\left(p_{1}+p_{2}\right)-\left(p_{1}-p_{2}\right)^{2}\right) .
\end{aligned}
$$


So in general:

$$
\begin{aligned}
& \left\langle Q_{i i}(t)\right\rangle=t\left(\left(p_{2 i-1}+p_{2 i}\right)-\left(p_{2 i-1}-p_{2 i}\right)^{2}\right), \\
& \left\langle Q_{i j}(t)\right\rangle=-t\left(p_{2 i-1}-p_{2 i}\right)\left(p_{2 j-1}-p_{2 j}\right) .
\end{aligned}
$$

Finally, substituting the values (3.10), (3.11) into equations (3.7) and (3.8), we receive expressions for shape parameters $A$ and $S$ of the system:

$$
\begin{aligned}
& A\left(\left\{p_{i}\right\}\right)=\left(\sum_{i=1}^{3}\left(p_{2 i-1}+p_{2 i}-\left(p_{2 i-1}-p_{2 i}\right)^{2}\right)^{2}\right. \\
& -\sum_{\substack{i, j=1 \\
i<j}}^{3}\left[\left(p_{2 i-1}+p_{2 i}-\left(p_{2 i-1}-p_{2 i}\right)^{2}\right)\left(p_{2 j-1}+p_{2 j}-\left(p_{2 j-1}-p_{2 j}\right)^{2}\right)\right. \\
& \left.\left.+3\left(p_{2 i-1}-p_{2 i}\right)^{2}\left(p_{2 j-1}-p_{2 j}\right)^{2}\right]\right) /\left(\sum_{i=1}^{6} p_{i}-\sum_{i=1}^{3}\left(p_{2 i}-p_{2 i-1}\right)^{2}\right)^{2}, \\
& S\left(\left\{p_{i}\right\}\right)=\left(1 2 \prod _ { i = 1 } ^ { 3 } \left(\left(p_{2 i-1}+p_{2 i}-\left(p_{2 i-1}-p_{2 i}\right)^{2}\right)\right.\right. \\
& +2 \sum_{i=1}^{3}\left(p_{2 i-1}+p_{2 i}-\left(p_{2 i-1}-p_{2 i}\right)^{2}\right)^{3} \\
& -3 \sum_{\substack{i, j=1 \\
i \neq j}}^{3}\left(p_{2 i-1}+p_{2 i}-\left(p_{2 i-1}-p_{2 i}\right)^{2}\right)^{2}\left(p_{2 j-1}+p_{2 j}-\left(p_{2 j-1}-p_{2 j}\right)^{2}\right) \\
& \left.-9 \sum_{\substack{i, j=1 \\
i \neq j}}^{3}\left(p_{2 i-1}+p_{2 i}-\left(p_{2 i-1}-p_{2 i}\right)^{2}\right)\right)\left(p_{2 i-1}-p_{2 i}\right)^{2}\left(p_{2 j-1}-p_{2 j}\right)^{2} \\
& \left.-18 \sum_{\substack{i, j, k=1 \\
i \neq j \neq k}}^{3}\left(p_{2 i-1}+p_{2 i}-\left(p_{2 i-1}-p_{2 i}\right)^{2}\right)\right)\left(p_{2 j-1}-p_{2 j}\right)^{2}\left(p_{2 k-1}-p_{2 k}\right)^{2} \\
& \left.-54 \prod_{i=1}^{3}\left(p_{2 i-1}-p_{2 i}\right)^{2}\right) /\left(\sum_{i=1}^{6} p_{i}-\sum_{i=1}^{3}\left(p_{2 i-1}-p_{2 i}\right)^{2}\right)^{3} .
\end{aligned}
$$

The quantities (3.12), (3.13) are universal in the sence that they do not depend either on time or on number of particles, and appear to be the functions only of transition probalities $p_{i}$. Note, that in real experiments and in computer simulations, one would obtain the quantities given by (3.12), (3.13) as the asymptotical values in the limits of large enough number of particles and long enough time of spreading process $(t \rightarrow \infty, N \rightarrow \infty)$.

The above scheme can be generalized to the case, when one has random values $p_{i}(\vec{r})$ (with $\left.\vec{r}=\{x, y, z\}\right)$ at different sites of the lattice, taken from some distribution $\rho\left(\left\{p_{i}(\vec{r})\right\}\right)$, so that the averaged value $\overline{p_{i}}$ is given by

$$
\overline{p_{i}}=\sum p_{i}(\vec{r}) \rho\left(\left\{p_{i}(\vec{r})\right\}\right) .
$$

Such a situation may occur due to the action of local random fields or presence of structural defects, occupying some sites of the lattice. Dealing with such systems that display randomness of structure, one should perform the double averaging for all quantities of interest [12, 24]: first, the configurational averaging over all possible 
trajectories of particles according to (2.2), and then the averaging over all different realizations of disorder according to:

$$
\overline{\langle\mathcal{O}\rangle}=\sum \rho\left(\left\{p_{i}(\vec{r})\right\}\right)\langle\mathcal{O}\rangle=\mathcal{O} \overline{P\left(t,\left\{n_{i}^{a}\right\}\right)} .
$$

Here, $\overline{P\left(t,\left\{n_{i}^{a}\right\}\right)}$ is the distribution function (2.1) averaged over disorder realizations of disorder:

$$
\overline{P\left(t,\left\{n_{i}^{a}\right\}\right)}=\frac{t !}{\prod_{i=1}^{5} n_{i}^{a} !\left(t-\sum_{i=1}^{5} n_{i}^{a}\right) !} \sum \rho\left(\left\{p_{i}(\vec{r})\right\}\right) \prod_{i=1}^{6} \prod_{k=1}^{n_{i}^{a}} p_{i}\left(\vec{r}_{k}\right)
$$

where $p_{i}\left(\vec{r}_{k}\right)$ denotes a probability to jump in direction $i$ for a walker which is located at site $\vec{r}_{k}$ of a lattice. In the case, when there are no correlations in distribution of $p_{i}(\vec{r})$ at different sites of the lattice, we can rewrite in last expression:

$$
\sum \rho\left(\left\{p_{i}(\vec{r})\right\}\right) \prod_{k=1}^{n_{i}^{a}} p_{i}\left(\vec{r}_{k}\right)=\prod_{k=1}^{n_{i}^{a}} \sum \rho\left(\left\{p_{i}(\vec{r})\right\}\right) p_{i}(\vec{r})=\bar{p}_{i}{ }^{n_{i}^{a}}
$$

so that all of the observables of interest in relations above can be obtained just by substituting $p_{i}$ by $\bar{p}_{i}$ in corresponding equations.

In the next section, we illustrate the results obtained by considering several model cases of invasion in anisotropic environment.

\section{EXAMPLES}

(1) In the most trivial isotropic case, when all $p_{i}$ are equal $\left(p_{i}=1 / 6\right)$, we have: $A=0, S=0$ (see Fig. 2(1)).

(2) Let us consider the case, when $p_{3}=1-p_{1}, p_{2}=p_{4}=p_{5}=p_{6}=0$ : the population is moving on the halfspace of $x y$ plane with non equal transition probabilities in $x$ and $y$ directions. It appears, that independently on the $p_{1}$ and $p_{3}$ values, we receive highly anisotropic, completely stretched configuration with $A=1, S=2$ (Fig. 2(2)).

(3) Next, let us assume $p_{1}=p_{2}, p_{3}=p_{4}=p_{5}=p_{6}=(1-2 p 1) / 4$ : moving along the $x$-axis is more (or less) probable, then in two remaining directions (Fig. 2(3)). In this case, on the basis of (3.12), (3.13) we obtain:

$$
\begin{aligned}
& A\left(p_{1}\right)=-3 p_{1}+9 p_{1}^{2}+\frac{1}{4}, \\
& S\left(p_{1}\right)=\frac{1}{4}\left(6 p_{1}-1\right)^{3} .
\end{aligned}
$$

Parameters $A$ and $S$ as functions of $p_{1}$ are shown in Figure 3. Note, that $p_{1}$ can vary in this case from 0 to $1 / 2$. At $p_{1}=\frac{1}{6}$, we restore the isotropic case (1) with $A=S=0$. Further increasing of $p_{1}$ leads to growing of anisotropy, until it reaches the final stage (configuration completely stretched along $x$ axis) at $p_{1}=p_{2}=\frac{1}{2}$, $p_{3}=p_{4}=p_{5}=p_{6}=0$.

(4) Finally, let us consider the most interesting case, when population of particles is spreading in environment with structural inhomogeneities (obstacles) in the form of parallel lines, randomly distributed in $x y$ plane and oriented along $z$ axis (see Fig. 4). Let $c$ be the concentration of lines $(0 \leq c \leq 1)$. From the point of view of each random walker, presence of lines does not prevent jumps in $z$ direction, but plays an essential role for movement in $x y$ plane. Really, for each lattice site, one of 4 nearest neighbors in $x y$ plane can be occupied with probability $c$ (belonging to the line) and is thus not allowed for random walker. The probability, that $k$ nearest neighbors $(0 \leq k \leq 4)$ are occupied, is given by Bernoulli formula for binomial probability distribution:

$$
\rho(k)=\frac{4 !}{k !(4-k) !} c^{k}(1-c)^{4-k} .
$$




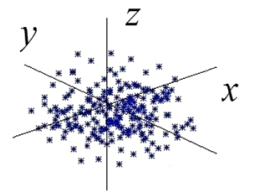

(1)



(2)

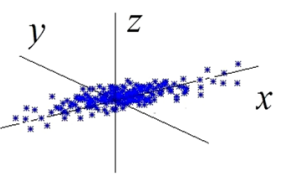

(3)

FIGURE 2. Instantaneous configurations of population of $N=100$ random walkers on a lattice after $t=2000$ steps, results of computer simulations. (1) Isotropic case with $p_{i}=1 / 6$. The values of shape parameters $A=0, S=0$. (2) Moving on the half-space of $x y$ plane with $p_{1}=0.4, p_{3}=0.6, p_{2}=p_{4}=p_{5}=p_{6}=0$. Maximal anisotropic state with $A=1, S=2$. (3) The case, when moving in $x$ direction is more probable, than in others: $p_{1}=p_{2}=0.4$, $p_{3}=p_{4}=p_{5}=p_{6}=0.05$. The values of shape parameters: $A=0.49, S=0.68$.

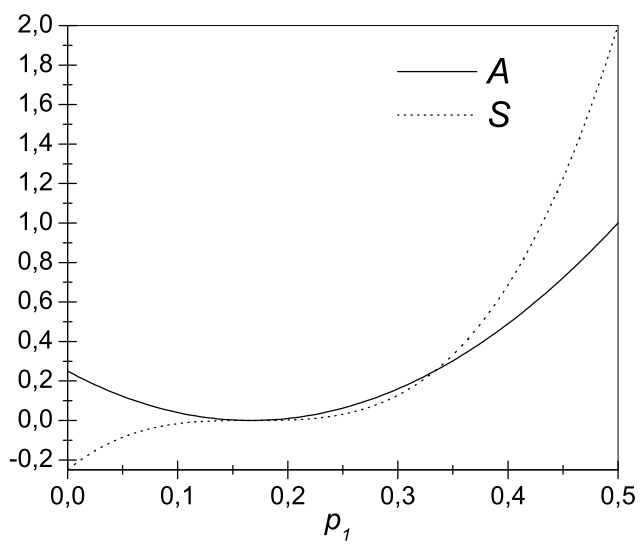

Figure 3. Shape parameters $A$ (as given by Eq. (4.1)) and $S$ (given by (4.2)) as functions of probability $p_{1}=p_{2}\left(\right.$ whereas $\left.p_{3}=p_{4}=p_{5}=p_{6}=0\right)$.

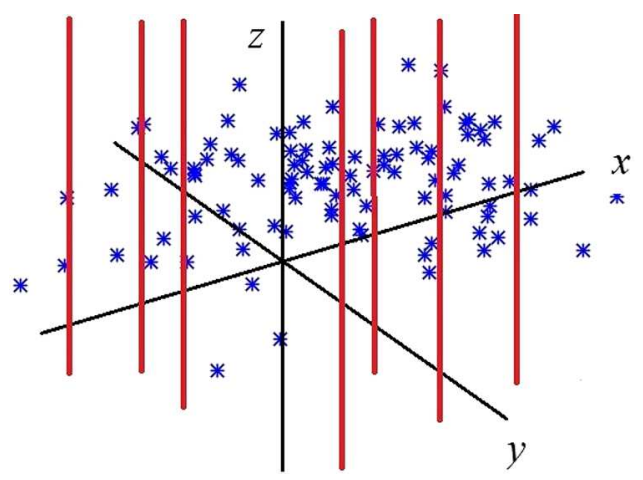

FIgURE 4. Schematic presentation of an instantaneous configuration of population spreading on a lattice in the presence of array of lines (tubes) oriented along $z$ axis.

The given problem thus essentially differs from examples above, where transition probabilities were spatiallyindependent constants $p_{i}$. Here, due to randomness of defects distribution in space, at each lattice site we observe one of possible $p_{i k}=1 /(6-k)$ with corresponding probabilities $\rho(k)$ given by (4.3). Namely, for $i=5,6$ 




Figure 5. Averaged transition probabilities of moving in $z$ direction (as given by Eq. (4.4)) and in $x y$ plane (Eq. (4.5)) as functions of concentration $c$ of oriented lines.

(transition probabilities in $z$ direction) taking into account equation (3.15) we have:

$$
\begin{aligned}
\overline{p_{i}}= & \sum_{k=0}^{4} p_{i k} \rho(k)=\sum_{k=0}^{4} \frac{1}{6-k} \rho(k)=\frac{1}{6}(1-c)^{4}+\frac{4}{5} c(1-c)^{3} \\
& +\frac{3}{2} c^{2}(1-c)^{2}+\frac{4}{3} c^{3}(1-c)+\frac{1}{2} c^{4}, \quad i=5,6 .
\end{aligned}
$$

The corresponding transition probabilities $p_{i k}$ in $x y$ planes are smaller by the factor $(4-k) / 4$ due to the fact, that $k$ jumps are forbidden by presence of defects, so that:

$$
\begin{aligned}
\overline{p_{i}}= & \sum_{k=0}^{4} \frac{4-k}{4} \frac{1}{6-k} \rho(k)=\frac{1}{6}(1-c)^{4}+\frac{3}{5} c(1-c)^{3} \\
& +\frac{3}{4} c^{2}(1-c)^{2}+\frac{1}{3} c^{3}(1-c), \quad i=1, \ldots, 4 .
\end{aligned}
$$

As expected, we have:

$$
\sum_{i=1}^{6} \overline{p_{i}}=1 .
$$

The functions (4.4) and (4.5) are presented graphically in Figure 5. At $c=0$, we restore the pure isotropic case when all $\overline{p_{i}}=1 / 6$. Increasing of $c$ leads to separation of these quantities: transition probabilities of moving in $z$ direction are growing, whereas corresponding values in $x y$ plane are gradually tending to zero. Approaching the very large values of $c$ around 1 , when there are practically no possibility of moving in $x y$ plane, $\overline{p_{5}}$ and $\overline{p_{6}}$ reach the limiting values of $1 / 2$. Note also, that $c=1$ (fully occupied lattice) has no physical meaning in our case, since there is no possibility for particles to move at all.

Let us recall, that in problem under consideration we need to perform the averaging of function (3.16) over distribution of $p_{i}(\vec{r})$. Note, that since the structural defects are randomly distributed in $x y$ plane, there is in principle no correlations in $p_{i}(\vec{r})$ and $p_{j}\left(\overrightarrow{r^{\prime}}\right)$ with $i, j=1, \ldots, 4$ at different sites of the lattice. However, since defects have a form of lines oriented along $z$ axis, the correlations occur in corresponding $p_{i}(x, y, z)$ and $p_{j}(x, y, z \pm 1)$ (namely, always $p_{i}(x, y, z)=p_{j}(x, y, z \pm 1)$ with $\left.i, j=5,6\right)$. So, when the walking particle is 


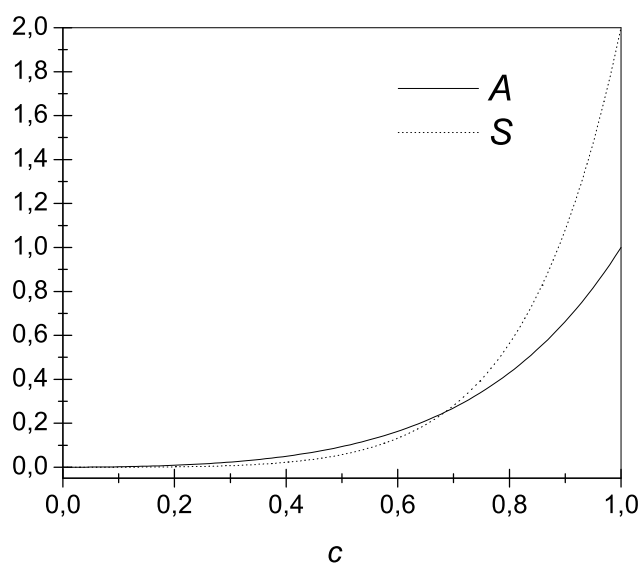

Figure 6. Shape parameters $A$ (given by Eq. (4.9)) and $S$ (Eq. (4.10)) as functions of concentration $c$ of oriented lines.

performing a series of $l$ consequent steps in direction $z$, we have in (3.16):

$$
\begin{aligned}
& \sum_{k=0}^{4} \rho(k) \prod_{m=1}^{l} p_{i}\left(\vec{r}_{m}\right)=\sum_{k=0}^{4} \rho(k) p_{i k}^{l}=\sum_{k=0}^{4} \rho(k)\left(\frac{1}{6-k}\right)^{l}=(1-c)^{4}\left(\frac{1}{6}\right)^{l} \\
& +4 c(1-c)^{3}\left(\frac{1}{5}\right)^{l}+6 c^{2}(1-c)^{2}\left(\frac{1}{4}\right)^{l}+4 c^{3}(1-c)\left(\frac{1}{3}\right)^{l}+c^{4}\left(\frac{1}{2}\right)^{l}
\end{aligned}
$$

whereas in case of no correlations between $p_{i}(\vec{r})$ one would have:

$$
\sum_{k=0}^{4} \rho(k) \prod_{m=1}^{l} p_{i}\left(\vec{r}_{m}\right)=\prod_{m=1}^{l} \sum_{k=0}^{4} \rho(k) p_{i k}={\overline{p_{i}}}^{l},
$$

with $\overline{p_{i}}$ given by equation (4.4). Taking into account, that $c$ is a small parameter, and comparing expressions (4.7) and (4.8) at fixed values of $c$ and $l$, we found that difference between them is of order of magnitude $10^{-3}$ at $l=2$ but becomes negligibly small at large $l$. To avoid difficulties with taking into account all possibilities of series of subsequent steps in $z$ direction, in what follows we will make use of assumption (4.8), leading to simple substituting $p_{i}$ by $\bar{p}_{i}$ in final expressions for observables. We stress however, that due to this assumption our results are rather of qualitative character and are reliable at small values of concentration $c$.

Substituting (4.4), (4.5) into expressions for the shape parameters (3.12), (3.13), we find corresponding expressions:

$$
\begin{aligned}
A= & \frac{1}{100} c^{2}\left(c^{6}+4 c^{5}+10 c^{4}+20 c^{3}+25 c^{2}+24 c+16\right), \\
S= & \frac{1}{500} c^{3}\left(c^{9}+6 c^{8}+21 c^{7}+56 c^{6}+111 c^{5}+174 c^{4}\right. \\
& \left.+219 c^{3}+204 c^{2}+144 c+64\right) .
\end{aligned}
$$

Expressions (4.9) and (4.10) as functions of $c$ are plotted in Figure 6. As expected, the asymmetry of shape is increasing with growing of the concentration of structural defects: the configuration of spreading population becomes more and more elongated in $z$ direction. Thus, the presence of an array of oriented fibers (tubes) leads to considerable spatial organization of invading population in environment. 


\section{Conclusions}

In the present work, we developed the simple mathematical model of population of agents, spreading in heterogeneous environment which is characterized by spatial anisotropy. It can be related to numerous processes, encountered in biology and medicine, such as chemotaxis or gravitaxis, the cell invasion in extracellular environment, migrations of living organisms in oriented habitats with non-homogeneous spatial distributions of resources, etc.

The presence of orientational factors in environment induces significant changes in geometrical shape of instantaneous configuration and spatial organization of spreading population. The motivation of the present study was to analyze the asphericity $A$ and prolateness $S$ as shape characteristics of instantaneous configuration of a group of particles, spreading in anisotropic environment with preferred orientations. For this purpose, we studied a system of non-interacting $N$ random walkers, spreading on an infinite $d=3$-dimensional lattice, starting with initial very dense configuration ("drop"). The transition probabilities $p_{i}(i=1, \ldots, 6)$ in different directions are assumed to be non-equal (asymmetric). We expressed the shape characterisitcs in terms of fundamental random walk parameters and found the exact analytical values for the parameters, such as asphericity $A$ (Eq. (3.12)) and prolateness $S$ (Eq. (3.13)) as functions of transition probabilities $p_{i}$. These ideas can find their application in mathematical methods of analysis and classification of asymmetric shape pattern formation is processes of bacteria colonies dynamics in inhomogeneous substrates or in presence of gradient of a chemotactic factors, as well as cell migration in tissue matrices.

To illustrate the results obtained, we considered several model cases of oriented environment. Of particular interest is the case, when the array of lines (tubes) of parallel orientation is present in the system. This can serve as a model of extracellular matrix with collagen and elastin fibers or systems of oriented nanotube array in drug delivery implants. It is quantitatively shown, that presence of such objects leads to considerable spatial orientation and organization of invading population.

\section{REFERENCES}

[1] T. Alarcón, H.M. Byre and P.K. Main, A cellular automaton model for tumour growth in inhomogeneous environment. J. Theor. Biol. 225 (2003) 257-274.

[2] W. Alt, Biased random walk model for chemotaxis and related diffusion approximation. J. Math. Biol. 9 (1980) $147-177$.

[3] A.R.A. Anderson, M.A.J. Chaplain, E.L. Newman, R.J.C. Steele and A.M. Thompson, Mathematical modelling of tumour invasion and metastasis. J. Theor. Med. 2 (2000) 129-154.

[4] P.R. Armsworth and L. Bode, The consequences of non-passive advection and directed motion for population dynamics. Proc. R. Soc. Lond. A 455 (1999) 4045-4060.

[5] P.R. Armsworth and J.E. Roughgarden, The impact of directed versus random movement on population dynamics and biodiversity patterns. Am. Nat. 165 (2005) 449-465

[6] J.A. Aronovitz and D.R. Nelson, Universal features of polymer shapes. J. Physique 47 (1986) 1445-1456.

[7] F. Belgacem and C. Cosner, The effects of dispersal along environmental gradients on the dynamics of populations in heterogeneous environments. Can. Appl. Math. Quar. 3 (1995) 379-397.

[8] E. Ben-Jacob and H. Levine, The artistry of nature. Nature 409 (2001) 985-986

[9] H.C. Berg, Motile Behavior of Bacteria. Phys. Today 53 (2000) 24-29.

[10] S. Bianchi, L. Biferale, A. Celani and M. Cencini, On the evolution of particle-puffs in turbulence. Eur. J. Mech. B Fluids 55 (2016) 324-329.

[11] M. Bishop and C.J. Saltiel, Polymer shapes in two, four, and five dimensions. J. Chem. Phys. 88 (1988) 3976-3980.

[12] R. Brout, Statistical mechanical theory of a random ferromagnetic system. Phys. Rev. 115 (1959) 824-835.

[13] R.S. Cantrell, C. Cosner and Y. Lou, Movement toward better environments and the evolution of rapid diffusion. Math. Biosci. 204 (2006) 199-214.

[14] F.A.C.C. Chalub, P.A. Markowich, B. Perthame and C. Schmeiser, Kinetics models for chemotaxis and their drift-diffusion limits. Monatsh. Math. 142 (2014) 123-141.

[15] A. Chauviere, T. Hillen and L. Preziosi, Modeling cell movement in anisotropic and heterogeneous network tissues. Netw. Heterogen. Media 2 (2007) 333-357.

[16] E.A. Codling, M.J. Plank and S. Benhamou, Random walk models in biology. J. R. Soc. Interface 5 (2008) $813-834$.

[17] E. Cristiani, P. Frasca and B. Piccoli, Effects of anisotropic interactions on the structure of animal groups. J. Math. Biol. 62 (2011) 569-588.

[18] S. Dewhirst and F. Lutscher, Dispersal in heterogeneous habitats: thresholds, spatial scales, and approximate rates of spread. Ecology 90 (2009) 1338-1345. 
[19] R.B. Dickinson, A generalized transport model for biased cell migration in an anisotropic environment. J. Math. Biol. 40 (2000) 97-135.

[20] R.B. Dickinson, S. Guido and R.T. Tranquillo, Biased cell migration of fibroblasts exhibiting contact guidance in oriented collagen gels. Ann. Biomed. Eng. 22 (1994) 342-356.

[21] H.W. Diehl and E. Eisenriegler, Universal shape ratios for open and closed random walks: exact results for all $d$. J. Phys. A: Math. Gen. 22 (1989) L87-L91.

[22] Y. Dolak and C. Schmeiser, Kinetic models for chemotaxis: Hydrodynamic limits and spatiotemporal mechanics. J. Math. Biol. 51 (2005) 595-615.

[23] C. Di Franco, E. Beccari, T. Santini, G. Pisaneschi and G. Tecce, Colony shape as a genetic trait in the pattern-forming Bacillus mycoides. BMC Microbiol. 2 (2002) 1-15.

[24] V.J. Emery, Critical properties of many-component systems. Phys. Rev. B 11 (1975) 239-247.

[25] P. Friedl and K. Wolf, Tumour-cell invasion and migration: diversity and escape mechanisms. Nat. Rev. 3 (2003) $362-374$.

[26] G. Gaspari, J. Rudnick and A Beldjenna, The shapes of open and closed random walks: a l/d expansion. J. Phys. A: Math. Gen. 20 (1987) 3393-3414.

[27] N.A. Hill and D.P. Häder, A biased random walk model for the trajectories of swimming micro-organisms. J. Theor. Biol. 186 (1997) 503-526.

[28] T. Hillen, $\mathrm{M}^{5}$ mesoscopic and macroscopic models for mesenchymal motion. J. Math. Biol. 53 (2006) 585-616.

[29] T. Hillen and K.J. Painter, Transport and Anisotropic Diffusion Models for Movement in Oriented Habitat, in Dispersal, Individual Movement and Spatial Ecology. Vol 2071 of Lecture Notes in Mathematics. Springer, Berlin (2013) 177-222.

[30] R.W. van Kirk and M.A. Lewis, Integrodifference models for persistence in fragmented habitats. Bull. Math. Biol. 59 (1997) $107-137$.

[31] W. Kuhn, Über die Gestalt fadenförmiger Moleküle in Lösungen, Kolloid-Z. 68 (1934) 2-15.

[32] D. Losic, M. Aw, A. Santos, K. Gulati and M. Bariana, Titania nanotube arrays for local drug delivery: recent advances and perspectives. Expert Opin. Drug Deliv. 12 (2015) 103-127.

[33] N.H. Mendelson, Helical growth of Bacillus subtilis: a new model of cell growth. Proc. Natl. Acad. Sci. USA 73 (1976) $1740-1744$

[34] Z. Mokhtari et al., Automated characterization and parameter-free classification of cell tracks based on local migration behavior. PLoS One 8 (2013) e80808.

[35] T.J. Pedley and J.O. Kessler, A new continuum model for suspensions of gyrotactic micro-organisms. J. Fluid Mech. 212 (1990) 155-182.

[36] K.J. Painter, Modelling cell migration strategies in the extracellular matrix. J. Math. Biol. 58 (2009) $511-543$.

[37] C.S. Patlack, Random walk with persistence and external bias. Bull. Math. Biophys. 15 (1953) 311-338.

[38] R. Rudner, O. Martsinkevich, W. Leung and E.D. Jarvis, Classification and genetic characterization of pattern forming Bacilli. Mol. Microbiol. 27 (1998) 687-703.

[39] J. Rudnick and G. Gaspari, The aspherity of random walks. J. Phys. A 19 (1986) L191-L194.

[40] B. Salhi and N. Mendelson, Patterns of gene expression in Bacillus subtilis colonies. J. Bacteriol. 175 (1993) $5000-5008$

[41] M. Sarris et al., Inammatory chemokines direct and restrict leukocyte migration within live tissues as glycan-bound gradients. Curr. Biol. 22 (2012) 2375-2382.

[42] S.J. Sciutto, Study of the shape of random walks. J. Phys. A Math. Gen. 27 (1994) 7015-7034.

[43] J.A. Shapiro, The use of Mudlac transposons as tools for vital staining to visualize clonal and non-clonal patterns of organization in bacterial growth on agar surfaces. J. Gen. Microbiol. 130 (1984) 1169-1181.

[44] J.A. Shapiro, Scanning electron microscope study of Pseudomonas putida colonies. J. Bacteriol. 164 (1985) 1171-1181.

[45] J.A. Shapiro, The significance of bacterial colony patterns. Bio Essays 17 (1995) 597-607.

[46] M.F. Shlesinger and B. West, Random Walks and their Applications in the Physical and Biological Sciences. Vol. 109 of AIP Conference Proceedings. AIP, New York (1984).

[47] K. Šolc and W.H. Stockmayer, Shape of a Random-Flight Chain. J. Chem. Phys. 54 (1994) 2756-2757.

[48] K. Šolc, Shape of a Random Flight Chain. J. Chem. Phys. 55 (1971) 335-344.

[49] R.V.V. Vincent and N.A. Hill, Bioconvection in a suspension of phototactic algae. J. Fluid Mech. 327 (1996) $343-371$.

[50] Q. Wang et al., Recent advances on smart $\mathrm{TiO}_{2}$ nanotube platforms for sustainable drug delivery applications. Int. J. Nanomed. 12 (2017) 151-165.

[51] B.P. Yurk, Homogenization analysis of invasion dynamics in heterogeneous landscapes with differential bias and motility. $J$. Math. Biol. 77 (2017) 27-54.

[52] M. Weber et al., Interstitial dendritic cell guidance by haptotactic chemokine gradients. Science 339 (2013) $328-332$.

[53] G. Wei and X. Zhu, Shapes and sizes of arbitrary random walks at $O\left(1 / d^{3}\right)$ II. Asphericity and prolateness parameters. Physica A 237 (1997) 423-440.

[54] A. Zakharchenko, N. Guz, A.M. Laradji, E. Katz and S. Minko, Magnetic field remotely controlled selective biocatalysis. Nat. Catal. 1 (2018) 73-81. 